\title{
PHYSICAL ACTIVITY AND OLDER ADULTS
}

\author{
B. Tumanova* \\ Sport Department, Sofia University “St. Kliment Ohridski”, Sofia, Bulgaria
}

\begin{abstract}
Physical activity among the older population of Bulgaria is a matter related to health prevention, improving the quality of life and recovery after illness. In this work, we present data from a study held with people aged 65-80 years, citizens of Sofia city. Our goal was to determine the place of physical exercise in their daily lives and the role of sports teacher for the formation of motivation in them for leading an active lifestyle. The analysis shows lack of information on the wide variety of motor activities suitable for this age group as well as low level of motivation for sport activities. We conclude that it is necessary to provide sources of information to the people of the third age, which will educate them about the benefits of the active way of life and what possibilities for access to various sports activities are there. Medium intensity workout or a daily walk outdoors would help not only to improve their fitness level but also to maintain the necessary mental health and social activity.
\end{abstract}

Key Words: physical exercises, motor activity, health, information, elderly people

\section{INTRODUCTION}

Old age is an important stage of one's life. Specialists recommend physical exercises during this period to healthy individuals whose medical examinations have not registered any issues. Such individuals are directed to appropriate physical exercises that correspond to their needs. Appropriate physical exercises have proven their indispensability throughout one's entire lifespan. Regardless of whether old people are active, inactive, ill, disabled or not - it is of vital importance for their good quality of life that they are physically active (4). Conducted tastings have determined that a diverse and balanced diet combined with mild physical exercises contribute to the old's desired physical shape; build up psychological health and emotional comfort. The physical exercises include swimming, gymnastics, various kinds of sport games and tourism.

Sports increases quality of life, improves health, reduces households' spending and brings about tolerance (1). The place of the sports pedagogue is crucial in motivating the elderly to get

\footnotetext{
*Correspondence to: Assoc. Prof. Boryana

Tumanova Phd, Sport Department, Sofia University

"St. Kliment Ohridski”, E-mail:

b_tumanova@yahoo.com, mobile: 359888969724
}

involved in various sorts of physical exercises, in providing health information and in providing sufficient support for the formation of a healthy lifestyle. In her research, the scholar Rangelova emphasizes the growing need for physical education teachers that could adapt physical exercises to the specific needs of the trained (5).

\section{EXPOSITION}

There is no universal exercise program for the elderly. Physical exercises are adjusted to each person's individual characteristics, eventual illnesses and the role of sport activities in their lifestyle. Specialists recommend that, during exercises, the main rules of therapeutic gymnastics are followed:

- at the beginning, exercises should last 15-20 minutes, and eventually be extended to 25-30 minutes;

- exercises should be conducted calmly with slow to medium temp

- pressure should be increased gradually;

- physical exercises should be implemented only after a medical consultation

- exercises should be practiced regularly

- before exercises a few minutes of walking are recommended in order to warm up the organism. 
For healthy people it is recommended that the weekly physical activity is with moderate intensity. The time devoted to physical exercises is evenly spread throughout the week. Important is the period dedicated to the preparation (warming up) before the actual training. It should be started with warming-up exercises before the main ones so as to allow the body to prepare itself. Appropriate is the initiation of exercises for building up the back muscles, the inclusion of large muscle groups provide the following: walking, dancing and swimming. Their regular practice helps the activity of the cardio muscle, the lungs, and the bone-joint apparatus. Adequate are moderate aerobic exercises which last 15-30 minutes. The temp of physical pressure is increased slowly and gradually. In this way the muscles are toned up. Parallel to physical exercises, liquids must be taken. The sport pedagogue should inform for the required appropriate outfit - the clothing must be comfortable, produced from natural materials.

In the case of indisposition, sport practicing is not recommended and fatigue should be avoided. Exercises must be terminated at signs of pain.

Through the motor activity the central neuro system is impacted as the processes and reflexes in the cerebral cortex are mobilized and the general body tonus is raised, and general and local influence on thee organs and systems of the elderly organism is exercised.

In the preliminary research among the elderly it was found that the willing to practice physical exercises are anxious whether that would not
TUMANOVA B.

harm their health and whether there exists the opportunity to do sports in an organized manner and under the supervision of a specialist.

According to G. Ignatov, the motivation issue is central in the training activity. Without knowing the motives, it is impossible to predict the behavior of the trainees and it to be successfully managed (3). Hristova in her own research specifies that the education of the elderly with regards to the merits of physical exercising would significantly increase their motivation for sport engagement (2). The reason for the implementation of the present study is the registered need for an adequate policy which builds up the motivation of the old part of the population for their participation in various physical exercises, forming the physical and psychological health of our populace.

\section{METHODOLOGY OF THE RESEARCH}

Research among people aged from 65 to 80 was conducted.

Aim: to be studied the participation of the examined contingent in various physical exercises and the place of the sport pedagogue in forming motivation in these people for an active lifestyle.

A representative sample, randomly generated and comprising 105 individuals has been composed.

The data are gathered anonymously and voluntarily through an online form in the period 01.10.2018-30.03.2019.

The results are graphically represented

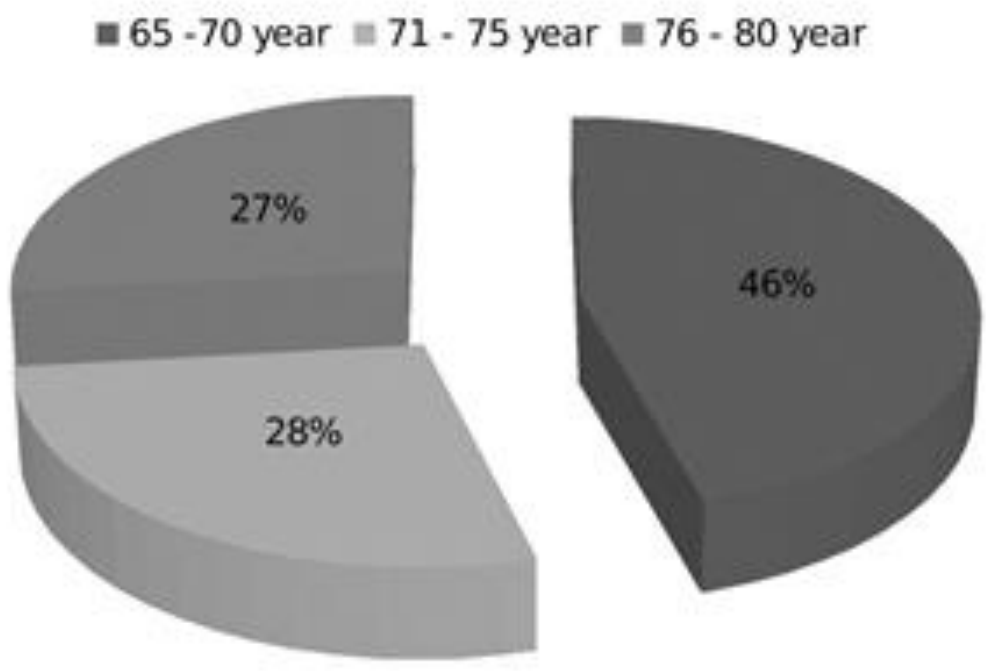

Figure 1. Age range of the respondents 
The most active participation in the online form are the participants of age groups 65-70 (35 of them are women), while at the other two ranges the results are similar. The data from the study show that only $28(26 \%)$ of the respondents do physical exercises and take part in sport
TUMANOVA B.

activities. Of them 21 are women and 7 men. They are divided in the following age groupings:

- 65-70; 61\% (12 women and 5 men);

- $71-75 ; 18 \%$ (4 women and 1 man);

- $76-80 ; 21 \%$ (5 women and 1 man)

The motives for physical activity among the respondents are exhibited in the next graph.

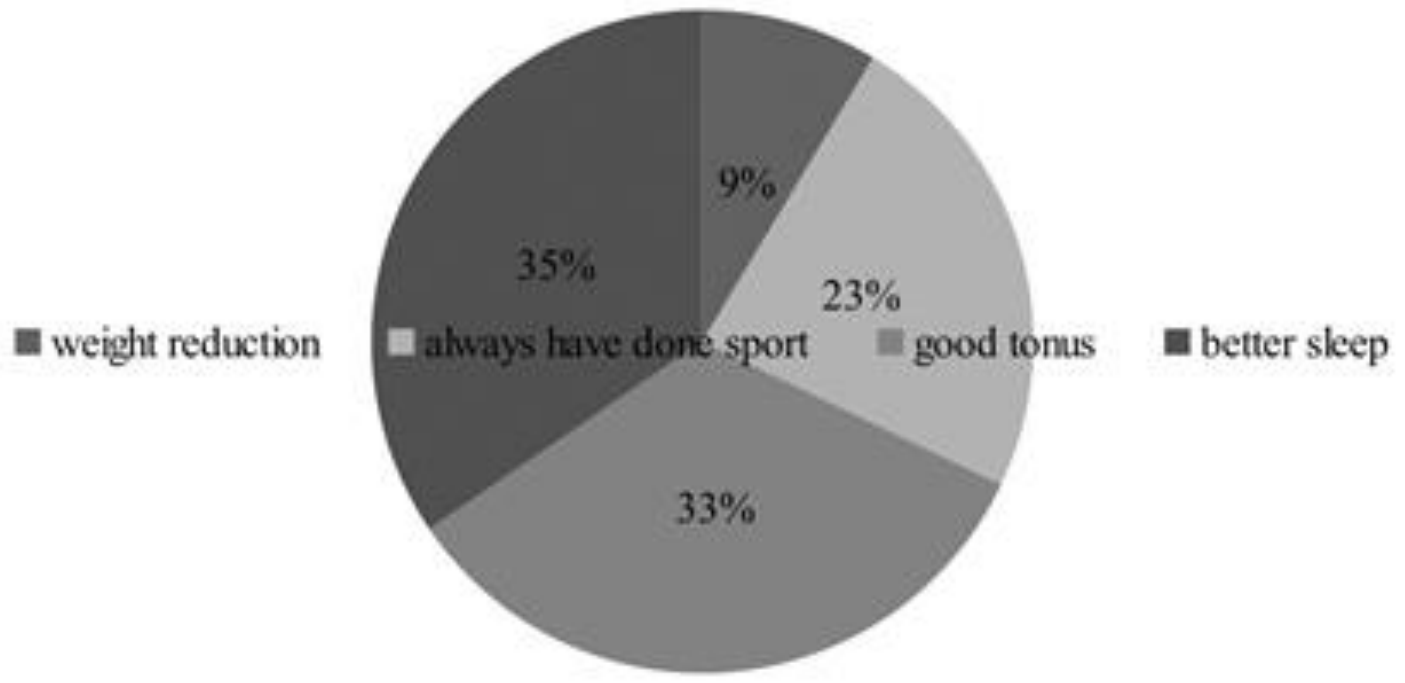

Figure 2. Factors motivating physical exercises

The group of studied individuals which take part in different physical exercises in order to improve their sleep is most numerous, followed by those for whom sport is a habit and lifestyle, and the smallest remains the group of those who sport to lose weight.
The data analysis of our research shows that the preferred kinds of motor activity among the elderly are tourism (42\%), folk dances $(28 \%)$, yoga $(18 \%)$ and swimming (12\%) - Figure 3.

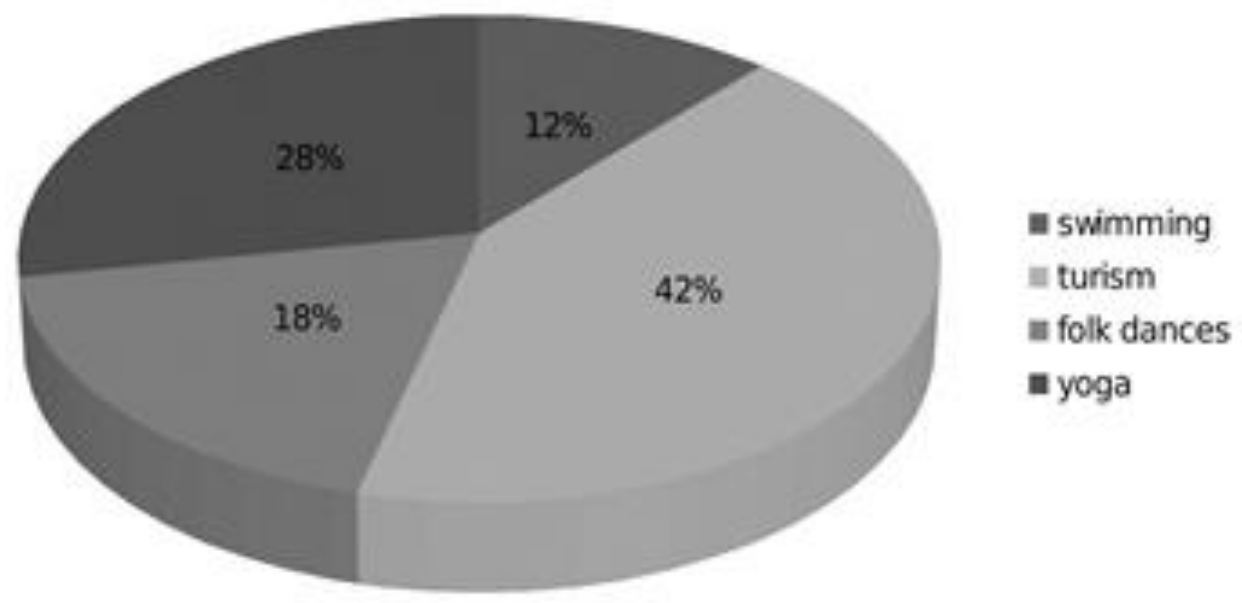

Figure 3. Preferred sport activities

Figure 4 illustrates the reasons which the respondents single out as demotivating them from physical activity which we group in the following way:

- The largest is the group of people who have financial problems stopping them from doing sport (52\%);
- The next group's individuals do not find sufficient information for the possibilities which could render them physically active in an organized and adequate for their age manner (34\%);

- $12 \%$ do not like to be physically active;

- $5 \%$ are obstructed by their health condition. 


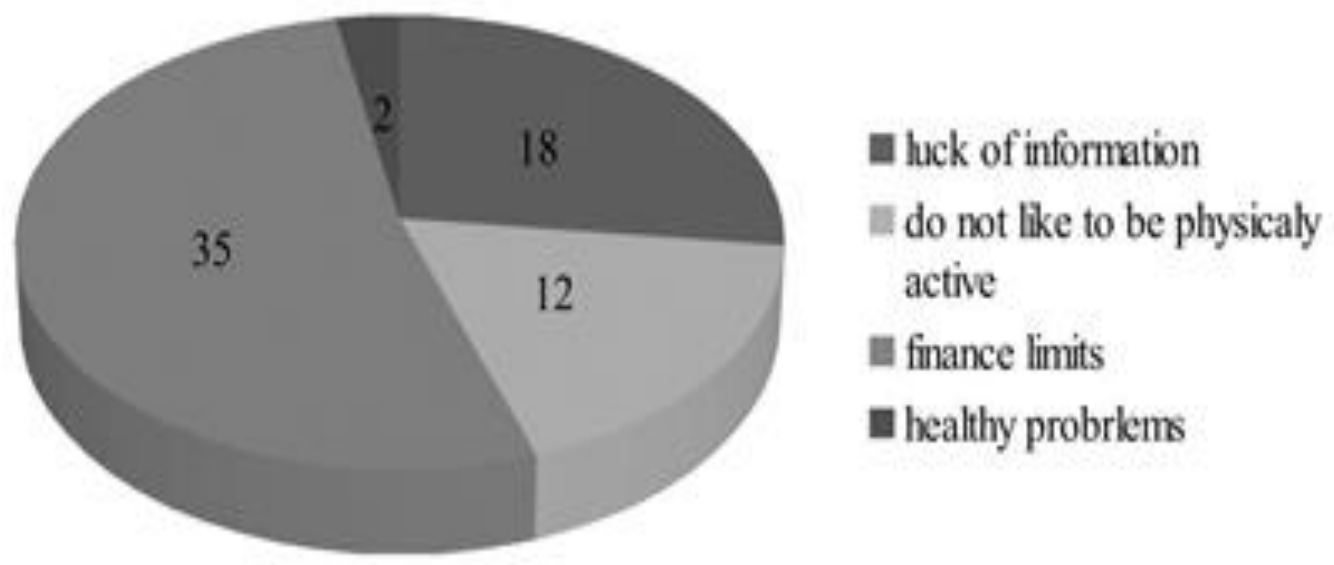

Figure 4. Reasons not to do sport

The data analysis from the conducted study demonstrates the necessity of health information for the physical regime of the elderly population in Bulgaria as prophylaxis for the physiological changes and various illnesses that happen during this period in the old organism care for a better social life and other issues related to health. The physical regime of the elderly must be conform to an adequate physical program. Each sport activity is connected to the using up of energy which should be compensated with the corresponding lifestyle regime and diet. The sport specialists ought to respond to the need for physical activity and training of the elderly in our country.

\section{IMPLICATIONS}

The results of the conducted study illustrate the lack of an adequate sport-pedagogue system of work with individuals from the examined age groups. The latter do not receive information for the need and use of physical activity. Physical exercises, moderate sport and daily outdoors walks bring about the effacement of various health problems that accompany old age. They help individuals not only be in great physical shape, but also impact on their psycho-emotional condition and socialization.

\section{REFERENCES}

1. Dochevska, Y., Lazarov, I., Zdravkov, I., Zankov, K., Ilieva. K., Martinova, D., Djobova, S. (2017). European week of sport - editions 2015 \& 2016. Good practices manual. ISBN 978-619-90776-27

2. Hristova, P (2015) A Study on the awareness of students and their expectations of the aerobics classes. "Modern Tendencies in Physical Education and Sport". ISSN 1314-2275, pp. 124-130, Sofia.

3. Ignatov, G., (2010). Motivational features for training sports dejnbost in football classes in SU. St.Kliment Ohridski “. The second international scientific conference. „Optimization and innovation in educational-training process“. Department of sport. University Publishing St. Kliment Ohridski“. ISSN 1314-2275, Sofia., 2010, pp. $34-45$

4. Niemiro, A., Huijsmans, K., Dobreva, I., Djobova, S. (2007). Never too old to be active. The joy of movement. THENAPA II. K.U.Leuven, Belgium

5. Rangelova, B. (2017). Swimming exercise suitable for babies. "European standards in the sports education" ISBN 978-619-728124-8, Vratsa. 